\title{
Microphthalmia-Associated Transcription Factor
}

\section{A Central Regulator of Pigment Epithelium-Derived Factor Controlling Human Melanoma Progression}

\author{
To the Editor-in-Chief:

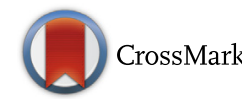 \\ Benilde Jiménez Cuenca, ${ }^{*}$ Asunción Fernández-Barral, ${ }^{*}$ and \\ José Luis $\mathrm{Orgaz}^{* \dagger}$
}

\author{
From the Department of Biochemistry,* Instituto de Investigaciones \\ Biomédicas "Alberto Sols", CSIC-UAM, Instituto de Investigación I+12, \\ Universidad Autónoma de Madrid, Madrid, Spain; and the Randall \\ Division of Cell and Molecular Biophysics Faculty of Life Sciences \& \\ Medicine ${ }^{\dagger}$ King's College London, London, United Kingdom
}

We read with great interest the article by Dadras et al ${ }^{1}$ in The American Journal of Pathology describing microphthalmiaassociated transcription factor (MITF) as a regulator of pigment epithelium-derived factor (PEDF) in human melanoma. Although we are pleased to see that the authors have confirmed our recent results, ${ }^{2}$ we feel that the authors overlooked a careful review of the concurrent literature on the biological role of PEDF during melanoma progression and the identification of MITF as a transcriptional regulator of PEDF.

First, Dadras et $\mathrm{al}^{1}$ state in their abstract that "PEDF expression and/or regulation during melanoma development have not been investigated previously."p.252 This statement is inaccurate, as our group ${ }^{3}$ and another ${ }^{4}$ have previously addressed this point. Specifically, by performing a highthroughput analysis of the data from molecular profiling studies of human melanoma cell lines, we demonstrated that PEDF is highly expressed in melanocytes and low aggressive melanoma cells but is lost in highly aggressive melanomas. ${ }^{3}$ Furthermore, we studied paired cell lines isolated from human metastatic lesions displaying extreme phenotypes and demonstrated that PEDF expression is restricted to the less aggressive counterparts. ${ }^{3}$ In mouse models of human melanoma metastases, we established that PEDF levels critically impact melanoma progression by gain and loss of function experiments (PEDF overexpression or silencing, respectively). ${ }^{3}$ The mention of a subsequent study identifying the mechanisms by which PEDF exerts its antimetastatic actions ${ }^{4}$ is also missing from the article by Dadras et al. ${ }^{1}$ Importantly, this study showed that PEDF is able to efficiently block two modes of melanoma invasion by suppressing ameboid morphology and mesenchymal proteolysis. ${ }^{4}$

Second, Dadras et al ${ }^{1}$ show that PEDF is a direct transcriptional target of MITF in human melanocytes and melanoma cells but overstate the novelty of their discoveries. We have recently demonstrated that PEDF expression positively correlates with MITF during human melanoma progression. $^{2}$ Of note, we showed that oncogene-induced senescence promotes down-regulation of PEDF and MITF in primary melanocytes, providing a likely explanation to the lack of PEDF and MITF expression in nevi described in our article ${ }^{2}$ and confirmed by Dadras et al. ${ }^{1}$ Furthermore, we identified three MITF regulatory sites in the first intron of SERPINF1 based on published ChIP-seq data ${ }^{5}$ that were further validated by an independent ChIP-seq experiment ${ }^{2}$ and reporter assays that revealed that at least two of the identified MITF-binding sites have functional activity. Importantly, our study also provided a functional connection between MITF and PEDF, which is critical for melanoma dissemination. ${ }^{2}$ Using an established zebrafish model of metastasis, we demonstrated that in vivo dissemination of human melanoma cells induced by MITF silencing is halted by PEDF sustained expression, ${ }^{2}$ providing solid evidence for the role of the MITF-PEDF axis in the control of melanoma biology.

In summary, we believe that the article published by Dadras et $\mathrm{al}^{1}$ lacks a complete description of the previous findings describing the biological role of PEDF and its regulation by MITF during human melanoma progression. We consider it important to highlight these references to the readers of the Journal.

Supported by Ministerio de Ciencia y Competitividad of Spain grant SAF-2011-24225.

Disclosures: None declared. 


\section{References}

1. Dadras SS, Lin RJ, Razavi G, Kawakami A, Du J, Feige E, Milner DA, Loda MF, Granter SR, Detmar M, Widlund HR, Horstmann MA, Fisher DE: A novel role for microphthalmia-associated transcription factor-regulated pigment epithelium-derived factor during melanoma progression. Am J Pathol 2015, 185:252-265

2. Fernández-Barral A, Orgaz JL, Baquero P, Ali Z, Moreno A, Tiana M, Gomez V, Riveiro-Falkenbach E, Cañadas C, Zazo S, Bertolotto C, Davidson I, Rodríguez-Peralto JL, Palmero I, Rojo F, Jensen LD, del Peso L, Jiménez B: Regulatory and functional connection of microphthalmia-associated transcription factor and anti-metastatic pigment epithelium derived factor in melanoma. Neoplasia 2014, 16:529-542

3. Orgaz JL, Ladhani O, Hoek KS, Fernández-Barral A, Mihic D, Aguilera O, Seftor EA, Bernad A, Rodríguez-Peralto JL, Hendrix MJ, Volpert OV, Jiménez B: Loss of pigment epithelium-derived factor enables migration, invasion and metastatic spread of human melanoma. Oncogene 2009, 28:4147-4161

4. Ladhani O, Sánchez-Martinez C, Orgaz JL, Jimenez B, Volpert OV: Pigment epithelium-derived factor blocks tumor extravasation by suppressing amoeboid morphology and mesenchymal proteolysis. Neoplasia 2011, 13:633-642

5. Strub T, Giuliano S, Ye T, Bonet C, Keime C, Kobi D, Le Gras S, Cormont M, Ballotti R, Bertolotto C, Davidson I: Essential role of microphthalmia transcription factor for DNA replication, mitosis and genomic stability in melanoma. Oncogene 2011, 30:2319-2332

http://dx.doi.org/10.1016/j.ajpath.2015.03.029

\section{Authors' Reply}

Soheil S. Dadras, ${ }^{*}$ Richard J. Lin, ${ }^{\dagger}$ and

David E. Fisher ${ }^{* \dagger}$

From the Cutaneous Biology Research Center and Department of Dermatology, * Massachusetts General Hospital, Harvard Medical School, Charlestown; and the Department of Pediatric Hematology/Oncology, ${ }^{\dagger}$

Melanoma Program in Medical Oncology, Boston, Massachusetts

We thank Dr. Benilde Jiménez Cuenca and colleagues for bringing to our attention the research by Fernandez-Barral et $\mathrm{al}^{1}$ reporting the regulatory and functional relationship imposed by microphthalmia-associated transcription factor (MITF) on pigment epithelium-derived factor (PEDF) in melanoma. We assure the authors that we were completely unaware of their publication, presumably because both manuscripts were likely under review at the same time. By the time our manuscript ${ }^{2}$ was accepted for publication (September 5, 2014), their article had only recently been published (June 2014).

Nevertheless, we are pleased that our in vitro findings were independently confirmed by another group of investigators. The two papers have indeed taken similar approaches in demonstrating the transcriptional control of PEDF by MITF in vitro and decreased PEDF expression during melanoma progression. In addition, our focus was more clinical by taking a cohort of 102 patients with welldefined and characterized melanocytic tumors to be investigated for PEDF expression by immunohistochemistry. We also correlated the expression of PEDF to the extent of measured angiogenesis in the melanoma biopsies, American Joint Committee on Cancer melanoma staging, and patient clinical outcome. Our finding of inverse correlation between PEDF expression and Breslow's depth of tumor invasion is a critical clinical parameter related to tumor progression. Depth of tumor invasion, along with ulceration and mitotic index, are currently the most important criteria to predict prognosis for melanoma patients. Thus we enthusiastically agree that Fernandez-Barral et al $^{1}$ have made significant contributions to the field. We also wish to highlight the value of extending the field by addition of the clinical correlations that our study provides.

\section{References}

1. Fernández-Barral A, Orgaz JL, Baquero P, Ali Z, Moreno A, Tiana M, Gomez V, Riveiro-Falkenbach E, Canadas C, Zazo S, Bertolotto C, Davidson I, Rodríguez-Peralto JL, Palmero I, Rojo F, Jensen LD, del Peso L, Jiménez B: Regulatory and functional connection of microphthalmia-associated transcription factor and anti-metastatic pigment epithelium derived factor in melanoma. Neoplasia 2014, 16: 529-542

2. Dadras SS, Lin RJ, Razavi G, Kawakami A, Du J, Feige E, Milner DA, Loda MF, Granter SR, Detmar M, Widlund HR, Horstmann MA, Fisher DE: A novel role for microphthalmia-associated transcription factor-regulated pigment epithelium-derived factor during melanoma progression. Am J Pathol 2015, 185:252-265

http://dx.doi.org/10.1016/j.ajpath.2015.05.002
Disclosures: None declared.

Current address of S.S.D., Department of Dermatology, University of Connecticut, Farmington, CT; of R.J.L., New York University Langone Medical Center, New York, NY. 